\title{
Synthesis and Antimicrobial Activities of some Antipyrine-Triazole-Conazoles
}

\section{Bazı Antipirin-Triazol-Konazollerin Sentezi ve Antimikrobiyal Aktiviteleri}

\section{Hacer BAYRAK}

Department of Chemistry and Chemical Processing Technology, Maçka Vocational School, Karadeniz Technical University, Trabzon, Turkey.

\section{A B ST R ACT}

tarting from antipyrine-triazole moiety some new kind of conazoles were synthesized. The synthesized compounds were screened for their antimicrobial activities against some test microorganisms. Among them compound 3 which is an reduction product of compound 2 showed very good antitubercular activity against Mycobacterium smegmatis compared with Streptomycin standard drug. Also among the conazoles compound $4 \mathrm{~b}$ and $4 \mathrm{c}$ showed good antitubercular activity.

\section{Key Words}

Antipyrine, azole, conazole, antimicrobial activity

\footnotetext{
ntipirin-triazol içeriğinden başlayarak bazı yeni konazoller sentezlendi. Sentezlenen bileşikler, bazı test mikroorganizmalarına karşı antimikrobiyal aktiviteleri açısından tarandı. Bunlar arasında, bileşik 2'nin bir indirgeme ürünü olan 3 nolu bileşik, Streptomisin standart ilacı ile karşılaştırıldığında Mycobacterium smegmatis'e karşı çok iyi bir antitüberküloz aktivite göstermiştir. Ayrıca konazoller arasında 4b ve 4c nolu bileşikler iyi bir antitüberküloz aktivite göstermiştir.
}

\section{Anahtar Kelimeler}

Antipirin, azol, konazol, antimikrobiyal aktivite.

Article History: Received: Jun 15, 2020; Revised: Aug 28, 2020; Accepted: Aug 28, 2020; Available Online: Sep 28, 2020 DOI: https://doi.org/10.15671/hjbc.669068

Correspondence to: H. Bayrak, Dep. Chem.and Chem. Processing Technol. Maçka Vocational School, Karadeniz Technical University, Trabzon, Turkey. E-Mail:h.bayrak@ktu.edu.tr 


\section{INTRODUCTION}

The synthesis of heterocyclic compounds containing five-membered rings has become increasingly important in recent years due to their pharmacological properties. Among them azole compounds are popular groups with its biological activities. Some examples of such compounds are Vorozole, Letrozole, Anostrozole and Itraconazole, which are currently used in cancer treatment and are conazole derivatives, contain an azole ring in their molecular structure [1, 2]. Luliconazole, Lanoconazole and Econazole drugs are still used as antifungal agents and they also contain an azole ring which are conazole analogues [3]. The antimicrobial resistance of pathogenic microorganisms to existing drugs has led to the need for synthesis of new drug derivatives. Therefore, an important field of study has been established for medicinal chemists. In recent years, compounds containing simple or complex triazole molecules have been synthesized as antitumor drugs [4-7]. For this purpose, many working groups have begun to design and synthesize compounds containing triazole rings bearing different functional groups $[8,9]$.

Antipyrine, the first pyrazole derivative compound, is still used today as anti-inflammatory, antipyretic, analgesic and antimicrobial drugs [10-13]. In this study, antimicrobial activities were obtained by synthesizing conazole derivative structures by synthesizing triazole compounds containing antipyrine nuclei. The synthetic methodology has been designed from Econazole drug mentioned at Figure 1.

\section{MATERIALS and METHODS}

\section{Chemistry}

All the chemicals used in this publication were obtained from Sigma-Aldrich and Merck without further purification. Melting points of the synthesized new compounds were obtained by using capillary tube in Stuart Brand SMP apparatus. Reaction times and purities were determined by thin layer chromatography. Infrared spectra were obtained by ATR apparatus on Perkin Elmer brand and 1600 serial IR devices. NMR spectra of the compounds were obtained from BRUKER AVENE ॥ $400 \mathrm{MHz}$ instrument in Karadeniz Technical University or Giresun University Central Research Laboratories. Mass spectra of the compounds were also obtained from Agilent Technologies branded 1260 Infinity 6230 TOF LC / MS model device at Giresun University Central Research Laboratory.

2-[2-(2,4-Dichlorophenyl)-2-oxaethyl]-5-\{[(1,5dimethyl-3-oxo-2-phenyl-2,3-dihydro-1 $\mathrm{H}$-pyrazol4-yl)amino]methyl\}-4-phenyl-2,4-dihydro-3H-1,2,4triazol-3-one (2)

To a solution of the individual compounds $1(10 \mathrm{mmol})$ in anhydrous ethanol was added a solution of metallic sodium $(10 \mathrm{mmol})$ in anhydrous ethanol and the mix-

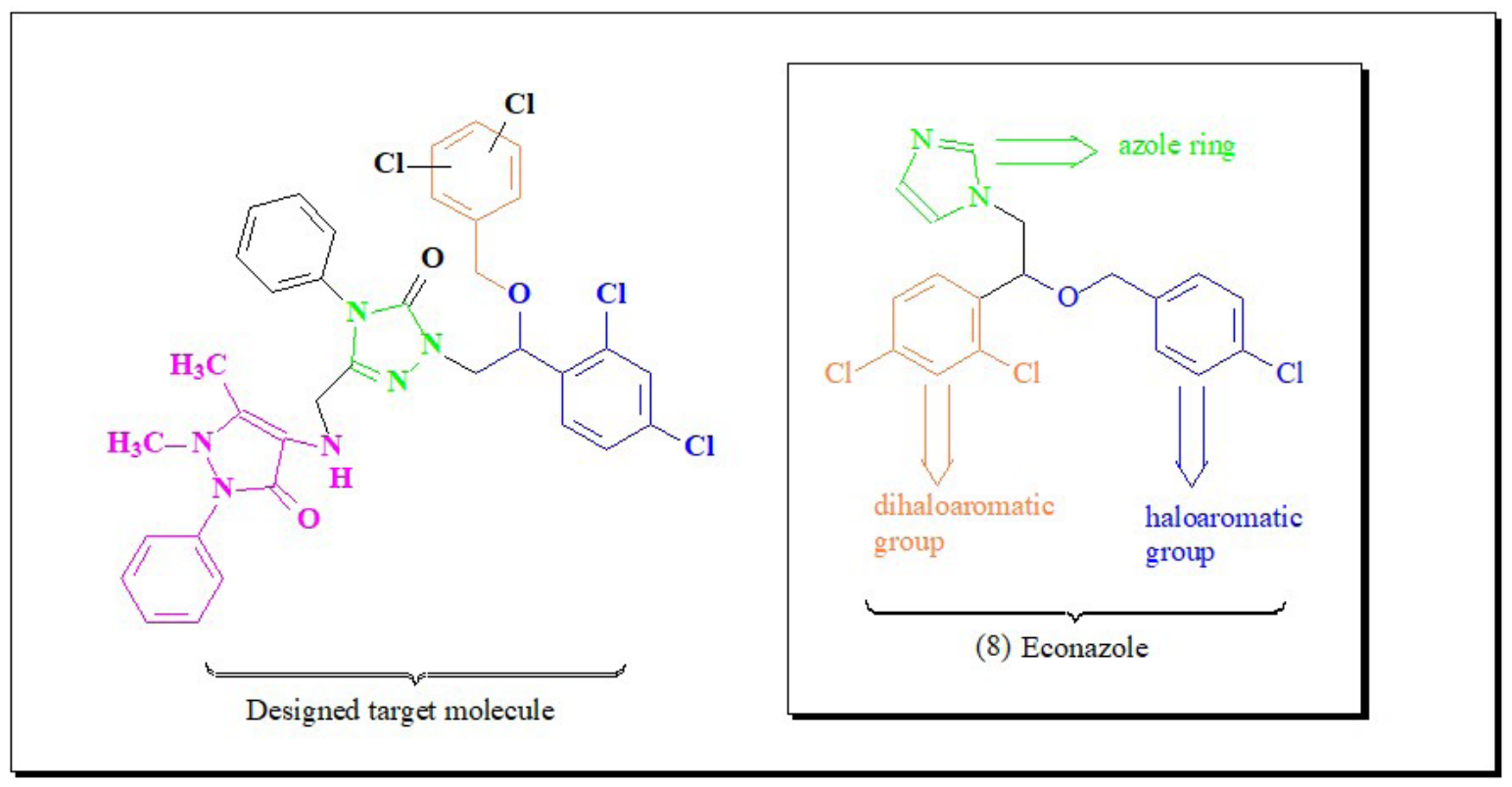

Figure 1.Designed target molecule compared with econazole. 
ture was refluxed for 2 hours. The mixture was cooled to room temperature and (2,4-dichlorophenyl)acetyl chloride $(10 \mathrm{mmol})$ was added and the mixture was refluxed for a further 14 hours. Water was added to the crude product obtained by evaporation of the solvent under reduced pressure, and the precipitated solid was filtered and purified by crystallization from a mixture of methanol: water (1: 1).

Yield 70 \%, m.p. $167-169{ }^{\circ} \mathrm{C}$.

FT-IR (U $\left.\mathrm{max}^{\prime} \mathrm{cm}^{-1}\right): 3294(\mathrm{NH}), 3091$ (aromatic $\left.\mathrm{CH}\right), 1708$ $(C=O), 1596(C=N)$.

${ }^{1} \mathrm{H}$ NMR (DMSO- $\left.d_{6}, \delta \mathrm{ppm}\right): 1.06-1.17\left(3 \mathrm{H}, \mathrm{m}, \mathrm{CH}_{3}\right), 2.21$ $\left(3 \mathrm{H}, \mathrm{d}, J=4.0 \mathrm{~Hz}, \mathrm{CH}_{3}\right), 3.04\left(2 \mathrm{H}, \mathrm{d}, J=8.0 \mathrm{~Hz}, \mathrm{CH}_{2}\right), 3.80$ $\left(2 \mathrm{H}, \mathrm{s}, \mathrm{CH}_{2}\right), 6.95(3 \mathrm{H}, \mathrm{s}, \mathrm{arH}), 7.26-7.50(7 \mathrm{H}, \mathrm{m}, \mathrm{arH}), 8.02$ $(3 \mathrm{H}, \mathrm{s}, \operatorname{arH}), 8.86(1 \mathrm{H}, \mathrm{d}, J=12.0 \mathrm{~Hz}, \mathrm{NH})$.

${ }^{13} \mathrm{C} \mathrm{NMR}$ (DMSO- $\left.d_{6}, \delta \mathrm{ppm}\right): 11.51\left(\mathrm{CH}_{3}\right), 36.25\left(\mathrm{CH}_{3}\right)$, $49.69\left(\mathrm{CH}_{2}\right), 66.06\left(\mathrm{CH}_{2}\right), 119.87$ (antipyrine $\left.\mathrm{C}-5\right)$, arC: [106.85 (CH), $107.16(\mathrm{C}), 111.20(\mathrm{CH}), 111.43(\mathrm{CH})$, $116.68(\mathrm{CH}), 118.63(\mathrm{C}), 118.95(\mathrm{C}), 119.92(\mathrm{CH}), 120.04$ $(\mathrm{CH}), 126.75(\mathrm{CH}), 131.30(\mathrm{CH}), 133.26(\mathrm{CH}), 133.44(\mathrm{CH})$, $133.67(\mathrm{CH}), 139.53(\mathrm{C}), 148.30(\mathrm{CH}), 150.75(\mathrm{C}), 151.81$ $(\mathrm{CH})$ ], 143.60 (antipyrine C-3), 145.46 (antipyrine C-4), 159.13 (triazole C-3), 163.34 (triazole C-5), 176.73 (C=O).

\section{2-[2-(2,4-Dichlorophenyl)-2-hydroxyethyl]-5-\{[(1,5- dimethyl-3-oxo-2-phenyl-2,3-dihydro-1H-pyrazol- 4-yl)amino]methyl\}-4-phenyl-2,4-dihydro-3H-1,2,4- triazol-3-one (3)}

$\mathrm{NaBH}_{4}$ (30 mmol) was added to a solution of the individual compound 2 (10 mmol) in absolute ethanol, and the mixture was refluxed for 10 hours. The solid precipitated by addition of water on the crude product. Then the solid was filtered and purified by crystallization from methanol: water (1: 1).

Yield $58 \%$, m.p. $144-146{ }^{\circ} \mathrm{C}$.

FT-IR $\left(U_{\text {max }} \mathrm{cm}^{-1}\right): 3299(\mathrm{NH}), 3133$ (aromatic $\left.\mathrm{CH}\right), 1581$ $(\mathrm{C}=\mathrm{N})$.

${ }^{1} \mathrm{H}$ NMR (DMSO- $\left.d_{6}, \delta \mathrm{ppm}\right): 1.17-1.24\left(3 \mathrm{H}, \mathrm{m}, \mathrm{CH}_{3}\right), 2.22$ $\left(3 \mathrm{H}, \mathrm{s}, \mathrm{CH}_{3}\right), 3.04\left(2 \mathrm{H}, \mathrm{s}, \mathrm{CH}_{2}\right), 3.40\left(2 \mathrm{H}, \mathrm{s}, \mathrm{CH}_{2}\right), 3.80(1 \mathrm{H}$, $\mathrm{s}, \mathrm{CH}), 4.13(1 \mathrm{H}, \mathrm{d}, \mathrm{J}=4.0 \mathrm{~Hz}, \mathrm{OH}), 7.27-7.48(13 \mathrm{H}, \mathrm{m}, \mathrm{arH})$, $9.62(1 \mathrm{H}, \mathrm{s}, \mathrm{NH})$.
${ }^{13} \mathrm{C}$ NMR (DMSO- $\left.d_{6}, \delta \mathrm{ppm}\right): 18.47\left(\mathrm{CH}_{3}\right), 19.35\left(\mathrm{CH}_{3}\right)$, $58.72\left(\mathrm{CH}_{2}\right), 59.30\left(\mathrm{CH}_{2}\right), 71.21(\mathrm{CH}), 139.48$ (antipyrine C-5), arC: [127.01 (CH), 127.14 (CH), $127.39(\mathrm{CH}), 127.46$ $(\mathrm{CH}), 127.49(\mathrm{CH}), 127.76(\mathrm{CH}), 128.52(\mathrm{CH}), 128.58$ $(\mathrm{CH}), 128.64(\mathrm{CH}), 128.66(2 \mathrm{CH}), 128.76(2 \mathrm{CH}), 140.43$ (C), 140.94 (C), 141.36 (C), 153.82 (2C)], 158.07 (antipyrine $\mathrm{C}-3$ ), 158.59 (antipyrine $\mathrm{C}-4), 159.23$ (triazole C-3), 169.46 (triazole C-5).

General Synthesis Method for Compounds 4a-4c

Separately to the solution of the compound $\mathbf{3 c}$ (10 $\mathrm{mmol}$ ) in THF, NaH (10 mmol) was added and was refluxed for 8 hours. The mixture cooled to room temperature and 2,4-dichlorobenzyl chloride, 2,6-dichlorobenzyl chloride or 4-chlorobenzyl chloride $(30 \mathrm{mmol}$ ) were added separately and refluxed for a further 10 hours. After removing the solvent under reduced pressure, an aqueous solution of $\mathrm{K}_{2} \mathrm{CO}_{3}$ was added and extracted with ethylacetate, the organic phase was dried over $\mathrm{Na}$ ${ }_{2} \mathrm{SO}_{4}$, filtered and the solvent was removed under reduced pressure. The resulting viscous product was purified by column chromatography (silica gel, n-hexane / ethylacetate, 7: 3).

2-[2-[(2,4-Dichlorobenzyl)oxy]-2-(2,4-dichlorophenyl) ethyl]-5-\{[(1,5-dimethyl-3-oxo-2-phenyl-2,3-dihydro1H-pyrazol-4-yl)amino]methyl\}-4-phenyl-2,4dihydro-3H-1,2,4-triazol-3-one (4a)

Yield $50 \%$.

FT-IR (U max $\left.^{\prime} \mathrm{cm}^{-1}\right): 3320(\mathrm{NH}), 3063$ (aromatic $\left.\mathrm{CH}\right), 1589$ $(\mathrm{C}=\mathrm{N})$.

${ }^{1} \mathrm{H}$ NMR (DMSO- $\left.d_{6}, \delta \mathrm{ppm}\right): 1.16-1.24\left(3 \mathrm{H}, \mathrm{m}, \mathrm{CH}_{3}\right), 1.84$ $\left(3 \mathrm{H}, \mathrm{s}, \mathrm{CH}_{3}\right), 2.09\left(2 \mathrm{H}, \mathrm{s}, \mathrm{CH}_{2}\right), 3.11\left(2 \mathrm{H}, \mathrm{s}, \mathrm{CH}_{2}\right), 5.12(1 \mathrm{H}, \mathrm{s}$, $\mathrm{CH}), 6.94(3 \mathrm{H}, \mathrm{d}, J=4.0 \mathrm{~Hz}, \mathrm{arH}), 7.26-7.65(13 \mathrm{H}, \mathrm{m}, \mathrm{arH})$, $9.52(1 \mathrm{H}, \mathrm{s}, \mathrm{NH})$.

${ }^{13} \mathrm{C}$ NMR (DMSO- $\left.d_{6}, \delta \mathrm{ppm}\right): 21.24\left(\mathrm{CH}_{3}\right), 36.74\left(\mathrm{CH}_{3}\right)$, $45.99\left(\mathrm{CH}_{2}\right), 47.98\left(\mathrm{CH}_{2}\right), 54.03\left(\mathrm{CH}_{2}\right), 74.32(\mathrm{CH}), 133.67$ (antipyrine C-5), arC: [107.56 (C), 108.05 (C), 119.71 (C), $127.28(\mathrm{CH}), 127.32(\mathrm{CH}), 127.45(\mathrm{CH}), 127.53(\mathrm{CH}), 127.68$ $(\mathrm{CH}), 127.75(\mathrm{CH}), 127.95(\mathrm{CH}), 128.01(\mathrm{CH}), 128.07$ $(\mathrm{CH}), 128.25(\mathrm{CH}), 128.44(\mathrm{CH}), 128.49(\mathrm{CH}), 128.69$ $(\mathrm{CH}), 128.71(\mathrm{CH}), 128.78(\mathrm{CH}), 128.80(\mathrm{CH}), 138.41(\mathrm{C})$, $138.70(C), 139.28$ (C), 139.87 (2C), 145.96 (C)], 151.42 
(antipyrine C-3), 152.13 (antipyrine C-4), 160.14 (triazole C-3), 160.99 (triazole C-5).

\section{2-[2-[(2,6-Dichlorobenzyl)oxy]-2-(2,4-} dichlorophenyl)ethyl]-5-\{[(1,5-dimethyl-3-oxo-2phenyl-2,3-dihydro-1H-pyrazol-4-yl)amino]methyl\}4-phenyl-2,4-dihydro-3H-1,2,4-triazol-3-one (4b)

Yield $48 \%$.

FT-IR (U max $\left.^{\prime} \mathrm{cm}^{-1}\right): 3317(\mathrm{NH}), 3063$ (aromatic $\left.\mathrm{CH}\right), 1596$ $(\mathrm{C}=\mathrm{N})$.

${ }^{1} \mathrm{H}$ NMR (DMSO- $\left.d_{6}, \delta \mathrm{ppm}\right): 1.15-1.26\left(3 \mathrm{H}, \mathrm{m}, \mathrm{CH}_{3}\right), 2.22$ $\left(3 \mathrm{H}, \mathrm{s}, \mathrm{CH}_{3}\right), 3.04\left(2 \mathrm{H}, \mathrm{d}, J=8.0 \mathrm{~Hz}, \mathrm{CH}_{2}\right), 3.35\left(2 \mathrm{H}, \mathrm{s}, \mathrm{CH}_{2}\right)$, $4.12(1 \mathrm{H}, \mathrm{d}, J=8.0 \mathrm{~Hz}, \mathrm{CH}), 7.26 .7 .30(3 \mathrm{H}, \mathrm{m}, \mathrm{arH}), 7.42-$ $7.56(13 \mathrm{H}, \mathrm{m}, \mathrm{arH}), 8.82(1 \mathrm{H}, \mathrm{s}, \mathrm{NH})$

${ }^{13} \mathrm{C}$ NMR (DMSO- $\left.d_{6}, \delta \mathrm{ppm}\right): 22.23\left(\mathrm{CH}_{3}\right), 36.24\left(\mathrm{CH}_{3}\right)$, $49.55\left(\mathrm{CH}_{2}\right), 50.01\left(\mathrm{CH}_{2}\right), 51.08\left(\mathrm{CH}_{2}\right), 79.74(\mathrm{CH}), 134.82$ (antipyrine $\mathrm{C}-5), \operatorname{arC}$ : [107.35 (C), $111.50(\mathrm{CH}), 111.72$ $(\mathrm{CH}), 119.75(\mathrm{C}), 125.20(\mathrm{CH}), 125.88(\mathrm{CH}), 127.55(\mathrm{CH})$, $128.13(\mathrm{CH}), 128.41(\mathrm{CH}), 129.36(\mathrm{CH}), 130.12(\mathrm{CH})$, $135.78(\mathrm{C}), 136.24(\mathrm{C}), 136.88(\mathrm{C}), 137.66(\mathrm{C}), 139.69(\mathrm{CH})$, $144.25(\mathrm{CH}), 145.99$ (C), 147.27 (2CH), 147.49 (C), 148.92 (2CH), $149.11(\mathrm{C})], 150.01$ (antipyrine C-3), 150.65 (antipyrine C-4), 152.15 (triazole C-3), 154.61 (triazole C-5).

2-[2-[(4-Chlorobenzyl)oxy-2-(2,4-dichlorophenyl) ethyl]-5-\{[(1,5-dimethyl-3-oxo-2-phenyl-2,3-dihydro1H-pyrazol-4-yl)amino]methyl\}-4-phenyl-2,4dihydro-3H-1,2,4-triazol-3-one (4c)

Yield $52 \%$.

FT-IR ( $\left.U_{\text {max }^{\prime}} \mathrm{cm}^{-1}\right): 3326(\mathrm{NH}), 3063$ (aromatic $\left.\mathrm{CH}\right), 1595$ $(\mathrm{C}=\mathrm{N})$.

${ }^{1} \mathrm{H}$ NMR (DMSO- $\left.d_{6}, \delta \mathrm{ppm}\right): 1.15-1.24\left(3 \mathrm{H}, \mathrm{m}, \mathrm{CH}_{3}\right), 1.78$ $\left(3 \mathrm{H}, \mathrm{s}, \mathrm{CH}_{3}\right), 2.09\left(2 \mathrm{H}, \mathrm{s}, \mathrm{CH}_{2}\right), 2.20\left(2 \mathrm{H}, \mathrm{s}, \mathrm{CH}_{2}\right), 3.04(1 \mathrm{H}$, $\mathrm{s}, \mathrm{CH}), 6.91(3 \mathrm{H}, \mathrm{d}, J=8.0 \mathrm{~Hz}, \operatorname{arH}), 7.21-7.25(7 \mathrm{H}, \mathrm{m}, \mathrm{arH})$, 7.37-7.57 (7H, m, arH), $10.53(1 \mathrm{H}, \mathrm{s}, \mathrm{NH})$.

$\left.{ }^{13} \mathrm{C} \mathrm{NMR} \mathrm{(DMSO}-d_{6}, \delta \mathrm{ppm}\right): 20.12\left(\mathrm{CH}_{3}\right), 39.52\left(\mathrm{CH}_{3}\right)$, $50.12\left(\mathrm{CH}_{2}\right), 51.87\left(\mathrm{CH}_{2}\right), 53.07\left(\mathrm{CH}_{2}\right), 73.52(\mathrm{CH}), 133.85$ (antipyrine $\mathrm{C}-5), \operatorname{arC}$ : [139.52 (CH), $140.25(\mathrm{CH}), 141.01$ $(\mathrm{CH}), 142.26$ (C), 143.85 (2CH), 144.03 (C), 144.96 $(2 \mathrm{CH}), 145.21(\mathrm{CH}), 146.83(2 \mathrm{CH}), 147.20(2 \mathrm{CH}), 148.10$ $(\mathrm{CH}), 149.10(2 \mathrm{CH}), 150.01(2 \mathrm{CH}), 151.71(\mathrm{C}), 152.33$ (C), 153.24 (C), 154.46 (C), 154.86 (C)], 155.38 (antipy- rine C-3), 156.64 (antipyrine C-4), 157.30 (triazole C-3), 158.11 (triazole C-5)

\section{Antimicrobial Activity / Sensitivity Studies}

The microdilution method was used to determine the dose value of the efficacy of the compounds effective in the agar well method. The amount of material is diluted to the lowest doses by serial dilutions and the same amount of microorganism is added to each diluent. With this test, the activity dose of the lowest amount of substance is determined.

\section{Minimal Inhibition Concentration (MIC) Method}

The MIC values have been determined as microgram / milliliter $(\mu \mathrm{g} / \mathrm{ml})$ [15]. For the determination of antimicrobial activity, liquid media were used for determining the antifungal activity of Mueller-Hinton liquid (MHB, pH.7.3) (Difco, Detroit, MI) and yeast extract liquid medium (YEG, pH 7.0) (Difco, Detroit). (MI). ELISA plates were used for micro-dilution tests and serial dilutions were made with $0.1 \mathrm{ml}$ of dissolved chemicals. McFarland 0.5 turbidity $\left(1 \times 10^{8} \mathrm{cfu} / \mathrm{mL}\right)$ from overnight cultures of inoculated microorganisms was prepared for reconstitution and diluted $1: 10$ and $0.005 \mathrm{ml}$ of microorganism (final assay concentration $5 \times 104 \mathrm{cfu} /$ well) was added to each well. Plates were incubated at $35^{\circ} \mathrm{C}$ for 16-24 hours under aerobic conditions. The MIC value was completely inhibited by the growth of the microorganism in the micro-dilution. Ampicillin $(10 \mu \mathrm{g})$, fluconazole for yeast $(5 \mu \mathrm{g})$, and standard solvent control were used as standard control drugs.

\section{RESULTS and DISCUSSION}

\section{Chemistry}

Compounds 1 (which was synthesized by our research group [14]) was reacted with 2,2,4-trichloroacetophenone to obtain compound $\mathbf{2}$ to form the aromatic carbonyl group. Subsequently, the reduction of the carbonyl group to alcohol with sodium borohydride was achieved to yield the corresponding compound $\mathbf{3}$. The intermediate starting materials in each series were reacted separately with 2,4-dichlorobenzyl chloride, 2,6-dichlorobenzyl chloride and 4-chlorobenzyl chloride respectively to synthesize the corresponding derivatives 4a-4c (Figure 2). 


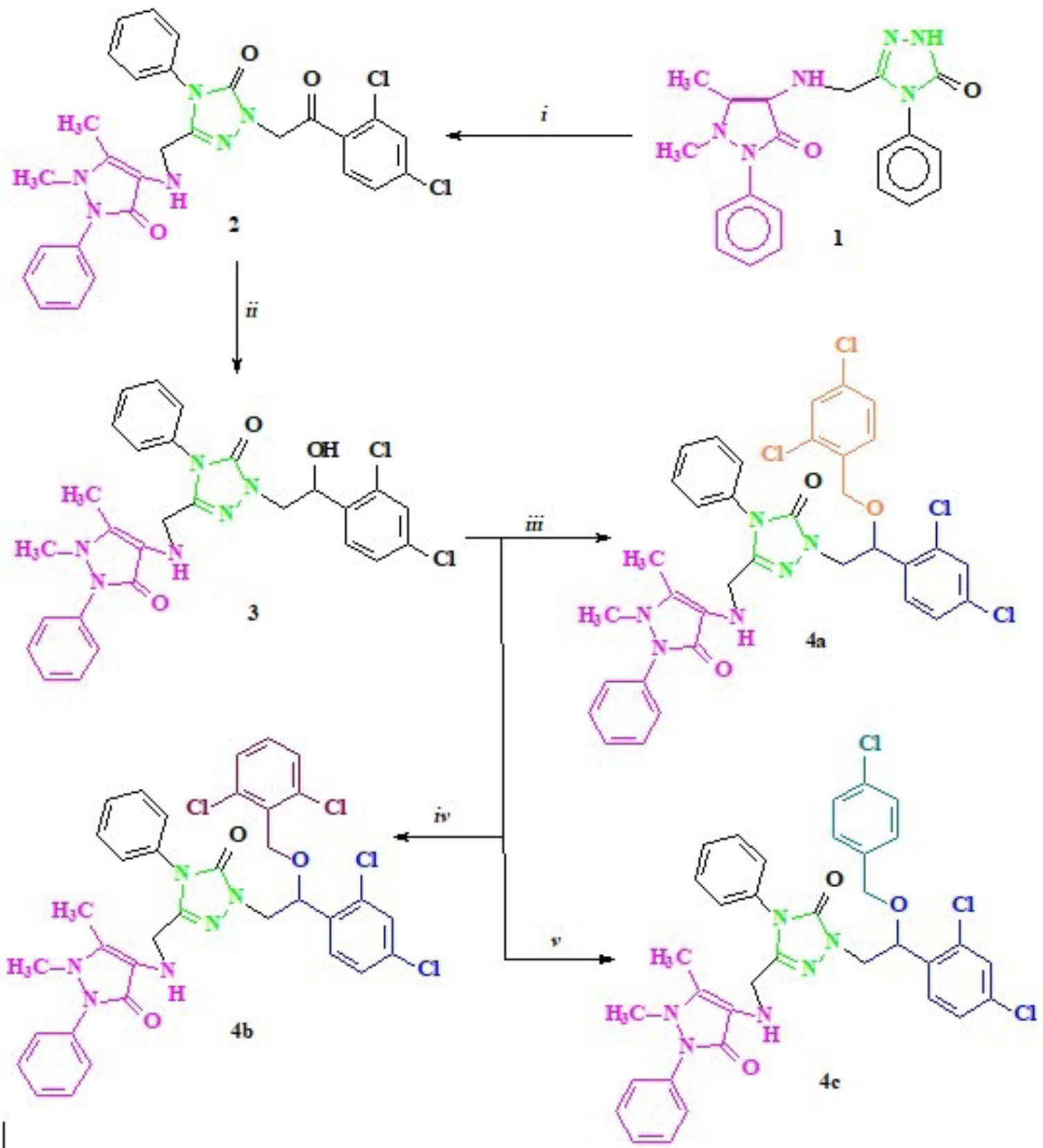

Figure 2. Synthetic route for compounds 1-4. Reagents: i. (-2,-4) $\mathrm{Cl}_{2} \mathrm{PhCOCH}_{2} \mathrm{Cl}$, ii. $\mathrm{NaBH}_{4}$, iii. $(-2,-4) \mathrm{Cl}_{2} \mathrm{PhCH}_{2} \mathrm{Cl}$, iv. $(-2,-6) \mathrm{Cl}_{2} \mathrm{PhCH}_{2} \mathrm{Cl}$, v. $(-4) \mathrm{ClPhCH}_{2} \mathrm{Cl}$.

The $-\mathrm{CH}$ and aromatic protons and carbon atoms proves the synthesized of reduction compound $\mathbf{3}$. Similarly, the increase in the number of aromatic rings of compounds $\mathbf{4 a - 4 c}$ of conazole derivatives have been identified in both proton and carbon NMR.

\section{Antimicrobial Activity}

In order to determine the in vitro activity of new compounds against specific bacteria, activity or susceptibility tests were performed. Diffusion and dilution methods were used as sensitivity tests. 
Table 1. Determination of Antimicrobial Activity of the new compounds by MIC method.

\begin{tabular}{|c|c|c|c|c|c|c|c|c|c|c|}
\hline \multirow{3}{*}{$\begin{array}{l}\text { Comp. } \\
\text { No }\end{array}$} & \multicolumn{10}{|c|}{ Microorganisms and Minimum Inhibition Concentrations (MIC) ( $\mu \mathrm{g} / \mathrm{mL})$} \\
\hline & \multicolumn{3}{|c|}{ Gram- Negative } & \multicolumn{4}{|c|}{ Gram-Positive } & \multirow{2}{*}{$\begin{array}{c}\text { No Gram } \\
\text { Ms }\end{array}$} & \multicolumn{2}{|c|}{ Yeast Fungi } \\
\hline & Ec & Yp & $\mathrm{Pa}$ & Sa & Ef & $\mathrm{Lm}$ & $\mathrm{Bc}$ & & $\mathrm{Ca}$ & Sc \\
\hline 2 & 125 & - & - & 125 & - & - & - & 125 & 125 & 125 \\
\hline 3 & 500 & - & - & 31,25 & - & - & 500 & 3,9 & - & - \\
\hline $4 a$ & 31,25 & 250 & - & - & 500 & - & - & 15,2 & - & - \\
\hline $4 b$ & 125 & - & - & - & - & - & - & 7,8 & - & - \\
\hline $4 c$ & 31,25 & - & 31,25 & 15,6 & 15,6 & 31,25 & 625 & 7,8 & 125 & 125 \\
\hline Amp. & 10 & 18 & $>128$ & 35 & 10 & 10 & 15 & & & \\
\hline Strep. & & & & & & & & 4 & & \\
\hline Flu. & & & & & & & & & $<8$ & $<8$ \\
\hline
\end{tabular}

It was found that compound 3, which is the reduction product of compound 2, showed excellent antitubercular activity against Mycobacterium smegmatis compared with Streptomycin standard drug. And compounds $4 \mathrm{~b}$ and $4 \mathrm{c}$, which are conazole analoges and contain 2,6-dichlorobenzyl and 4-chlorobenzyl group, showed good antitubercular activity. It is suprising that 4 a showed moderate antitubercular activity which also contain a 2,4-dichlorobenzyl group in its molecular structure different from compound $4 \mathrm{~b}$ and $4 \mathrm{c}$.

\section{CONCLUSION}

New kind of conazoles have been synthesized containing triazole and antipyrine moiety. Among them compounds $3,4 \mathrm{~b}$ and $4 \mathrm{c}$ showed good antitubercular activity against the standard drugs used (Table 1). All the compounds have been identified with such spectroscopic methods shown at the experimental section.

\section{Acknowledgments}

This work was supported by the Scientific research project of Karadeniz Technical University (KTU-BAP) [grant numbers 5289]. The author also thanks to Prof. Şengül Alpay Karaoğlu for the antitubercular activity studies.

\section{Conflict of Interest}

Authors declare that they have no conflict of interes.

\section{References}

1. B. Kolomodin-Hedman, G. Blomquist, E. Sikstrom, Mould exposure in museum personnel, Int. Arch. Occ. Env. Hea., 57 (1986) 321-323.

2. A.M. Krake, K.A.Worthington, K.M. Wallingford, K.F. Martinez, Evaluation of microbiological contamination in a museum, Appl. Occup. Environ. Hyg., 14 (1999) 499-509.

3. S. Sun, H. Lou, Y. Gao, P. Fan, B. Ma, W. Ge, Liquid chromatography-tandem mass spectrometric method for the analysis of fluconazole and evaluation of the impact of phenolic compounds on the concentration of fluconazole in Candida albicans, J. Pharmac. Biomed. Anal., 34 (2004) 1117-1124.

4. G.Verreck, K. Six, G. Van den Mooter, L. Baert, J. Peeters, M.E. Brewster, Characterization of solid dispersions of itraconazole and hydroxypropylmethylcellulose prepared by melt extrusion-part I. International Journal of Pharmaceutics, 251 (2003) 165-174.

5. M. Abastabar, A.M.S. Al-Hatmi, M.V. Moghaddam, G.S. de Hoog, I. Haghani, S.R. Aghili, Potent Activities of luliconazole, lanoconazole, and eight comparators against molecularly characterized fusarium species. antimicrob agents chemother, 62 (2018) e00009-18, /aac/62/5/e00009-18.

6. S.A.F. Rostom, M.A. Shalaby, M.A. El-Demellawy. Polysubstituted pyrazoles, part 5.11For part 4: see Ref. [18]. Synthesis of new 1-(4-chlorophenyl)-4-hydroxy-1Hpyrazole-3-carboxylic acid hydrazide analogs and some derived ring systems. A novel class of potential antitumor and anti-HCV agents, European J. Medicin. Chem., 38 (2003) 959-974.

7. G. Turan-Zitouni, Z.A. Kaplancikli, K. Erol, F.S. Kiliç, Synthesis and analgesic activity of some triazoles and triazolothiadiazines. II Farmaco, 54 (1999) 218-223.

8. G. Kolavi, V. Hegde, I.A. Khazi, P. Gadad, Synthesis and evaluation of antitubercular activity of imidazo[2,1-b][1,3,4] thiadiazole derivatives, Bioorgan. Medicin. Chem., 14 (2006) 3069-3080. 
9. B.S. Holla, B.K. Sarojini, B.S. Rao, P.M. Akberali, N.S. Kumari, V. Shetty, Synthesis of some halogen-containing 1,2,4-triazolo1,3,4-thiadiazines and their antibacterial and anticancer screening studies-Part I, II Farmaco, 56 (2001) 565-570.

10. J.N.N.S. Chandra, C.T. Sadashiva, C.V. Kavitha, K.S. Rangappa, Synthesis and in vitro antimicrobial studies of medicinally important novel $\mathrm{N}$-alkyl and $\mathrm{N}$-sulfonyl derivatives of 1-[bis(4-fluorophenyl)-methyl]piperazine, Bioorgan. Medicin. Chem., 14 (2006) 6621-6627.

11. P.P. Dixit, V.J. Patil, P.S. Nair, S. Jain, N. Sinha, S.K. Arora, Synthesis of 1-[3-(4-benzotriazol-1/2-yl-3-fluoro-phenyl)2-oxo-oxazolidin-5-ylmethyl]-3-substituted-thiourea derivatives as antituberculosis agents, European J. Medicin. Chem., 41 (2006) 423-428.

12. T.M. Abu-Elmaati, Reactions with Heterocyclic Amines: New Routes fort he Synthesis of Novel Azolo\{1,5- $\alpha$ ]Pyrimidine, some Pyridine, Pyran and Pyrazole Derivatives Containing The Antipyrine Moiety, Acta Chimica Slovenica, 49 (2002) 721-732.

13. M.T. El Sayed, M.A.M. El-Sharief, E.S. Zarie, N.M. Morsy, A.R. Elsheakh, A. Voronkov, Design, synthesis, anti-inflammatory activity and molecular docking of potential novel antipyrine and pyrazolone analogs as cyclooxygenase enzyme (COX) inhibitors, Bioorgan. Medicin. Chem. Lett., 28 (2018) 952957.
14. T. Bansal, M. Singh, G. Mishra, S. Talegaonkar, R.K. Khar, M. Jaggi, Concurrent determination of topotecan and model permeability markers (atenolol, antipyrine, propranolol and furosemide) by reversed phase liquid chromatography: Utility in Caco-2 intestinal absorption studies, J. Chromatogr. B, 859 (2007) 261-266.

15. N.A. El-Ghamaz, M.M Ghoneim, A.Z. El-Sonbati, M.A. Diab, A.A. El-Bindary, M.K. Abd El-Kader, Synthesis and optical properties studies of antipyrine derivatives thin films, J. Saudi Chemic. Soc., 21 (2017) S339-S348.

16. H. Bayrak, Y.U. Cebeci, S.A. Karaoğlu, Synthesis of novel antipyrine derivatives possessing excellent antimicrobial activities, ChemistrySelect, 6 (2019) 12906-12908.

17. G.L. Woods, Clinical and Laboratory Standards Institute (Wayne P. Susceptibility testing of mycobacteria, nocardiae, and other aerobic actinomycetes: approved standard. Wayne: Clinical and Laboratory Standards Institute; 2011. 